\title{
Complete Deafness
}

National Cancer Institute

\section{Source}

National Cancer Institute. Complete Deafness. NCI Thesaurus. Code C36194.

Total inability to hear sounds in one or both ears. 